\title{
Detail Preserving Fast Median Based Filter
}

\author{
Geeta Hanji, M.V.Latte \\ Research Scholar,J.N.T.U, Hyderabad and Associate Professor, E \& CE \\ Department, P.D.A Engineering College, Gulbarga, Karnataka, India. \\ E-mail:geetanjalipatil123@gmail.com \\ Principal, J.S.S.Academy for Technical Education, Bangalore, Karnataka, India. \\ E-mail:mvlatte@ rediffmail.com
}

\begin{abstract}
In this paper, a new nonlinear median based filtering technique is introduced for the enhancement of medical images that are highly contaminated by impulse noise. The proposed Detail Preserving Fast Median Filtering (DPFMF) technique is more effective in eliminating fixed value impulse noise and preserving the image details. The filter replaces a corrupted pixel by the median value of the fast median filter or by the value computed based on a unique criterion applied to the processed neighboring pixel values. The uncorrupted pixels are left undisturbed. Simulation studies show that the proposed filter can eliminate impulse noise of densities up to $80 \%$ while preserving the edges and fine details satisfactorily. The performance is evaluated by applying the proposed filter on different gray scale images and the superior results obtained in terms of subjective and objective evaluation are presented.
\end{abstract}

Keywords: Impulse Noise, Fast Median Filtering, Nonlinear Filter, Order Statistics Filter, Detail preservation, Image Enhancement.

\section{Introduction}

Impulse noise affects random signal positions of a digital image during acquisition stage when faulty sensors are used to acquire images in poor imaging conditions or during transmission when images are transmitted through faulty radio channels. Thus filtering is an essential need of image processing system, which addresses the estimation of image degradations and restoring the original image satisfactorily preserving the fine details intact. Several filtering techniques have been reported in the literature over the years, suitable for various 
applications. Nonlinear filtering techniques are preferred for cleaning the images which are degraded by impulse noise. These nonlinear filtering techniques take into account for nonlinear nature of the human visual system. Thus, the filters having good edge and image detail preservation properties are highly desirable for visual perception. The median filter and its variants are among the most commonly used filters for impulse noise removal. The non-linear Simple Median Filter (SMF) [3]- [5] is the first in its kind which sensibly replaces every pixel of the impulse corrupted image by considering the acute range of the strength of impulses with the mid-order statistic or the median of a set of pixels in the window sliding over each pixel of the image. The results though are significant than the linear counterparts; the median filters are indecisive in that all the pixels are replaced by the median to affect the reliability of the restored image. As an improvement did numerous variants of median filter evolved in the form of Weighted Median Filter (WMF), Center Weighted Median Filter (CWMF), Adaptive Center Weighted Median Filter (ACWMF), Adaptive Median Filter (AMF) [5]-[9] which weighed the pixels around each pixel differently according to the spatial positions of those neighboring pixels that an impulse-free pixel turns out to replace a particular pixel during the filtering operation. But these algorithms are also indecisive in that the filtering function was applied with no differentiation on all the corrupted and uncorrupted pixels of the digital image irrespective of the impulse noise ratio and the statistics of the image. So filtering algorithms with switching schemes are developed to detect and identify the spatial position of impulses in a distinct impulse detection phase. The prominent decision based impulse filter, the Progressive Switching Median Filter (PSMF) [10], though can produce results from images corrupted to slightly higher impulse ratios; its assurance that all impulses are correctly identified is meager since an optimal impulse detecting threshold suitable for a particular impulse noise ratio which varies for different window sizes could not be obtained by PSMF. Chan and Nikolova proposed a two-phase algorithm for high-density noise removal (RAMF) [11]. The main drawback of the method is that the processing time is very high because it uses a very large window of size. In addition, the switching schemes of certain decision based filtering algorithms could not differentiate between high frequency edge details of the corrupted digital image from high frequency impulses in view of the fact that they could not tune to various optimal impulse detection parameters suitable for varied impulse noise ratios and image statistics. Thus many filtering schemes are not adaptive in fixing a reliable neighborhood or other parameters to determine the correct impulsive position or an appropriate noise restorer suitable for that position of a particular impulsive environment. The Decision Based Algorithm (DBA) [12] is capable of removing impulse noise at noise densities as high as $80 \%$. A major drawback of this algorithm is streaking at higher noise densities. An Improved Decision -Based Algorithm for Impulse Noise Removal has been investigated [25]. In this method, a corrupted pixel is replaced by the average value of the already processed neighboring pixels inside the filter window. Although this filter suppresses 
impulse noise satisfactorily, it is found to exhibit inadequate performance in terms of preserving edges and fine details due to the averaging process involved in filtering. Robust Estimation Algorithms (REA) [13]-[16] are effective in highdensity impulse noise removal and detail preservation, but their computational complexity is higher. Many noise detection algorithms were proposed for impulse noise detection [17]-[23]. The Signal-Dependent Rank Ordered Mean (SD-ROM) [18] filter can remove impulse noise rather effectively, but when applied to images with Gaussian or mixed noise, it often produces a visually disappointing output similar to other median-based filters. This is because the rank-ordered mean gets corrupted in a high noise intensity window. The Directional Weighted Median (DWM) [9] filter uses an iterative filtering approach, and the detector is based on absolute differences within the filtering window. The estimation is done using an adaptive weighted median filter. For ensuring high accuracy of detection, iterative filtering is applied which takes a longer total processing time but removes more details with each iteration. The trilateral filter is based on RankOrder Absolute Difference (ROAD) [21] statistics for impulse noise detection. It has been especially designed for uniform impulse and Gaussian noise removal. The ROAD value could be false under the case that half of the pixels in the processing window are corrupted.

In this paper a new Detail Preserving Fast Median Filtering (DPFMF) Technique for removing fixed value impulse noise from the images is proposed. This filtering technique detects whether a pixel is noisy or noise-free based on minmax strategy. If the pixel is noise-free, the filtering window is moved forward to process the next pixel. On the other hand, if the pixel is a noisy one (either'0' or ' 255 '), then it is replaced by the median of Fast Median Filter (FMF) provided the median pixel value itself is not an impulse; otherwise, the pixel is replaced by the minimum or maximum of the already processed neighboring pixel value respectively in the filtering window. The proposed filter will be shown to exhibit better preservation of the edge details besides filtering out the noise sufficiently. The computational complexity involved in restoring the images is quite low and the processing time is also less because it uses small windows of size $3 \mathrm{X} 3$ and $5 \times 5$ depending on the amount of noise densities. The paper is organized as follows. Section 2 discusses the noise model and our new scheme proposed for impulse noise detection and elimination. The simulation results obtained by applying the filter on different images are presented in section 3 to illustrate its efficacy. The conclusions are summarized in section 4.

\section{Proposed Impulse Noise Detection and Restoration Scheme}

Impulse noise affects random signal positions of a digital image during acquisition stage when faulty sensors are used to acquire images in poor imaging conditions or during transmission when images are, transmitted through faulty 
radio channels. Of the various impulse noise models, the Salt and Pepper impulse that change the signals by replacing them with maximum and minimum values is modeled through equation (1) is better dealt by the proposed impulse filtering algorithm. Salt and pepper impulse model is described in equation (1) as follows:

$$
f(x)= \begin{cases}\frac{p}{2} & \text { for } x=0 \\ 1-p & \text { for } x=s(i, j) \\ \frac{p}{2} & \text { for } x=255\end{cases}
$$

where ' $p$ ' is the noise density

\subsection{Impulse Noise Detection Process}

The impulse noise detection is based on the assumption that a corrupted pixel takes a gray value which is significantly different from its neighboring pixels in the filtering window, whereas noise-free regions in the image have locally smoothly varying gray levels separated by edges. Our noise detection technique detects the impulse noise in the image using the min-max decision mechanism in which the corrupted and uncorrupted pixels in the image are detected by comparing the pixel value with the maximum ('255') and minimum (' 0 ') values in the selected window. If the pixel intensity lies between these minimum and maximum values, then it is an uncorrupted pixel and it is left undisturbed. If the value does not lie within the range, then it is marked as a corrupted pixel and needs to be restored.

\subsection{Restoration Process}

Corrupted pixels are restored by either the median pixel value of the fast median filter (provided that the median is not an impulse) or by the minimum or maximum of the already processed neighboring pixels in the current filtering window.

\subsection{Illustration of the proposed DPFMF technique}

Consider an image of size $\mathrm{M} \times \mathrm{N}$ having 8-bit gray scale pixel resolution. The steps involved in detecting the presence of an impulse $\&$ restoring the corrupted pixel value are described as follows:

Step 1) A two dimensional square filtering window of size $3 \times 3$ is slid over a corrupted image as shown in figure (1) below. In the figure ' 0 ' is the pixel under 
test and the pixels with gray values $162,159,163$ and 255 are the already processed pixels.

\begin{tabular}{l|c|c|c|c}
\cline { 2 - 4 } 161 & $\mathbf{1 6 2}$ & $\mathbf{1 5 9}$ & $\mathbf{1 6 3}$ & \multirow{2}{*}{63} \\
\cline { 2 - 4 } 167 & $\mathbf{2 5 5}$ & $\mathbf{0}$ & $\mathbf{2 5 5}$ & 255 \\
\cline { 2 - 4 } 164 & $\mathbf{2 5 5}$ & $\mathbf{2 5 5}$ & $\mathbf{2 5 5}$ & 255 \\
\cline { 2 - 4 } 165 & 0 & 255 & 255 & 255 \\
166 & 255 & 159 & 255 & 167
\end{tabular}

Fig 1: A segment of a noisy image with a 2-D square filtering window of size $3 \times 3$.

Step2) Minimum and maximum of the pixel values in the processing window are determined. In this case, the minimum and maximum values are 0 and 255 respectively.

Step3) Already processed pixels are sorted in the ascending order and the minimum and maximum of the sorted array are obtained. In this illustration, the sorted vector is $\{159,162,163,255\}$ and the minimum and the maximum pixel values are 159 and 255 respectively.

Step3) Median $\mathrm{M}_{\mathrm{f}}$ within the window is computed as follows:

The pixels inside the first, second and third row $R_{1}, R_{2}$ and $R_{3}$ are sorted in the ascending order as below:

$\mathrm{R}_{1}=\{159,162,163\}$

$\mathrm{R}_{2}=\{0,255,255\}$

$R_{3}=\{255,255,255\}$

The median $\mathrm{M}_{1}, \mathrm{M}_{2}$ and $\mathrm{M}_{3}$ are computed as below:

$\mathrm{M}_{1}=$ Median of $\mathrm{R}_{1}=162$

$\mathrm{M}_{2}=$ Median of $\mathrm{R}_{2}=255$

$\mathrm{M}_{3}=$ Median of $\mathrm{R}_{3}=255$

Fast median $\mathrm{M}_{\mathrm{f}}=$ median of $\{\mathrm{M} 1, \mathrm{M} 2$ and $\mathrm{M} 3\}$

In this case $M_{\mathrm{f}}=255$ which is noisy.

Step 4) If the central pixel lies between minimum and maximum values, then it is detected as an uncorrupted pixel and the pixel is left undisturbed. Otherwise, it is considered a corrupted pixel value. In the present case, the central pixel value ' $0 / 255$ ' does not lie between minimum and maximum values. Therefore, the pixel is detected as a corrupted pixel.

Step 5) The corrupted central pixel (either ' 0 ' or '255') is replaced by the fast median $\mathrm{M}_{\mathrm{f}}$ of the filtering window, provided $\mathrm{M}_{\mathrm{f}}$ value is not an impulse, 
otherwise it is replaced by either the minimum or maximum of the already processed neighboring pixel value in the filtering window respectively.

In this illustration the central pixel under test is ' 0 ', and the median $\mathrm{M}_{\mathrm{f}}$ itself is noisy. Hence the central pixel is replaced by the minimum of the processed pixels value, i.e. by ' 159 '.If the central pixel value is ' 255 ', and then it would have been replaced by the maximum of the processed pixel value, (i.e. by ' 255 ' in this example).

Then the window is moved to form a new set of values, with the next pixel to be processed at the centre of the window. This process is repeated until the last image pixel is processed.

The above impulse noise detection and filtering scheme is based on the following steps:

if $X_{\min }<X_{i, j}<X_{\max }$

$\left\{X_{i, j}\right.$ is a noiseless pixel;

no filtering is performed on $\mathrm{X}_{\mathrm{i}, \mathrm{j}}$ \}

else

$\left\{\mathrm{X}_{\mathrm{i}, \mathrm{j}}\right.$ is a noisy pixel;

Determine fast the median value $\mathrm{M}_{\mathrm{f}}$ \}

if median $\mathrm{M}_{\mathrm{f}} .0$ and median. 255

\{Median itself is noisy

Then

\{Processed neighbor substitution performed on $\mathrm{X}_{\mathrm{i}, \mathrm{j}}$ \}

$\left\{X_{i, j} .0\right.$ or 255$\}$

$\mathrm{X}_{\mathrm{i}, \mathrm{j}}=\operatorname{Min}($ Processed $)$ or Max $($ Processed $)$

end;

end;

where $X_{i, j}$ is the intensity of central pixel inside the filtering window, $\mathrm{X}_{\min ,} \mathrm{X}_{\max }$ and $\mathrm{M}_{\mathrm{f}}$ are the minimum, maximum and fast median pixel values in the filtering window of noisy image respectively. 


\section{Results and Discussion}

In this section, results are presented to illustrate the performance of the proposed DPFMF algorithm. Images are mixed with noise at different densities for evaluating the performance of the algorithm. Several grey scale images (viz; lena, cameraman, flower, baboon etc) are used for experimentation. A quantitative comparison is performed between several noise removal filters and the proposed DPFMF algorithm in terms of Peak Signal to Noise Ratio (PSNR) and the computational time. The noise model of equation (1) is simulated for the experimentation. A 3X3 and 5X5 windows are used for impulse densities in the range shown below in table 1 .

Table 1: Window sizes for impulse noise densities in the different ranges

\begin{tabular}{|l|c|}
\hline Noise density $\boldsymbol{P}$ & Window size \\
\hline $\mathrm{P}<40 \%$ & $3 \times 3$ \\
\hline $40 \% \leq \mathrm{P} \leq 90 \%$ & $5 \times 5$ \\
\hline
\end{tabular}

All the algorithms are implemented in MATLAB 7.5 on a PC equipped with AMD Athlon $64 \times 2$ Dual core processor of 2.71 GHZ Capacity and $1.75 \mathrm{GHZ}$ RAM memory.

The performance of the proposed filter is compared with several median based filtering techniques by applying them on Lena image of size 256 x 256, corrupted with various densities of impulse noise. DPFM filter has also been evaluated by applying it on several images of size $256 \times 256$. The objective measure used for quantitatively evaluating the performance of the filters is Peak Signal to Noise ratio (PSNR) and is defined as follows:

$$
P S N R=10 \log _{10}(255 \times 255 / M S E)
$$

where the mean square error $(M S E)$ is

$$
\operatorname{MSE}=\frac{1}{M N} \sum_{i=1}^{M} \sum_{j=1}^{N}\left(r_{i j}-x_{i j}\right)^{2}
$$

In the above equation $x(i, j)$ and $r(i, j)$ denote, respectively, the intensity of $(i, j)^{\text {th }}$ pixel of the original and filtered images. In order to prove the better performance of the proposed DPFMF, existing filtering techniques are experimented and compared with the proposed filter for visual perception and subjective evaluation on Lena grey scale image including The performance of the algorithm for various images at different noise levels from $10 \%$ to $90 \%$ is studied and results are shown in figure (1) and (2) for the Lena grey scale image. The first simulation compares 
the DPFMF algorithm with the Simple Median Filter (SMF), Progressive Switching Median Filter (PSMF), Adaptive Median Filter AMF and Rank Ordered Median Filter (RAMF), Decision Based Algorithm (DBA) and (REA). SMF replaces the current pixel by its median value irrespective of whether a pixel is corrupted or not. Therefore, its performance is poor which is evident from the results shown in table (2). Progressive Switching Median Filter (PSMF) [10] has slightly improved performance but its noise removing capacity is very poor at higher noise densities. AMF and RAMF exhibits improved performance but due to its adaptive nature the computation complexity is much higher which is evident from table (2). DBA has very good noise removing capability and good edge preservation at higher noise densities but it produces streaking at higher noise densities which is evident from visual perception shown in fig (1) and (2). REA has improved performance than DBA but its computational complexity is much higher as given in Table (2). The overall performance of the proposed DPFMF algorithm can be summarized as follows: The proposed algorithm removes noise effectively even at higher noise levels and preserves the edges and other fine details of the image. The proposed algorithm also exhibits lower computational time when compared to the other algorithms.

Table 2: PSNR of various filters for Lena image at different noise densities corrupted by salt \& pepper noise, where N.D. is the Noise Density.

\begin{tabular}{|c|c|c|c|c|c|c|c|c|c|}
\hline ND & SMF & PSMF & AMF & RAMF & DBA & TFROAD & REA & DWM & DPFMF \\
\hline 10 & 33.46 & 35.94 & 38.14 & 41.75 & 41.60 & 41.63 & 41.76 & 41.69 & 43.10 \\
\hline 20 & 29.04 & 32.38 & 35.94 & 37.56 & 37.48 & 38.49 & 38.20 & 37.35 & 38.51 \\
\hline 30 & 23.41 & 28.69 & 33.84 & 34.59 & 34.61 & 36.46 & 35.40 & 34.77 & 36.67 \\
\hline 40 & 18.95 & 25.10 & 31.97 & 32.19 & 32.30 & 34.77 & 33.44 & 32.63 & 34.83 \\
\hline 50 & 15.09 & 21.00 & 30.32 & 30.06 & 30.25 & 33.18 & 31.50 & 30.19 & 33.25 \\
\hline 60 & 12.24 & 16.71 & 28.58 & 27.78 & 28.11 & 31.52 & 29.43 & 28.24 & 31.87 \\
\hline 70 & 9.86 & 9.88 & 26.71 & 25.51 & 25.74 & 29.19 & 27.23 & 25.91 & 30.37 \\
\hline 80 & 7.93 & 7.98 & 25.13 & 22.98 & 22.94 & 25.89 & 24.70 & 23.49 & 28.49 \\
\hline 90 & 6.44 & 6.48 & 22.00 & 19.28 & 19.77 & 20.92 & 21.35 & 20.09 & 25.83 \\
\hline
\end{tabular}

Table 3: Computational time in seconds for filtering $80 \%$ salt \& pepper noise corrupted LENA (256X256) image for filters.

\begin{tabular}{|l|l|l|l|l|l|c|c|c|c|}
\hline FILTER & SMF & PSMF & AMF & RAMF & DBA & TFROAD & REA & DWM & DPFMF \\
\hline TIME & 0.18 & 6.5 & 6.46 & 8.16 & 9.55 & 8.55 & 9.28 & 9.24 & 5.36 \\
\hline
\end{tabular}



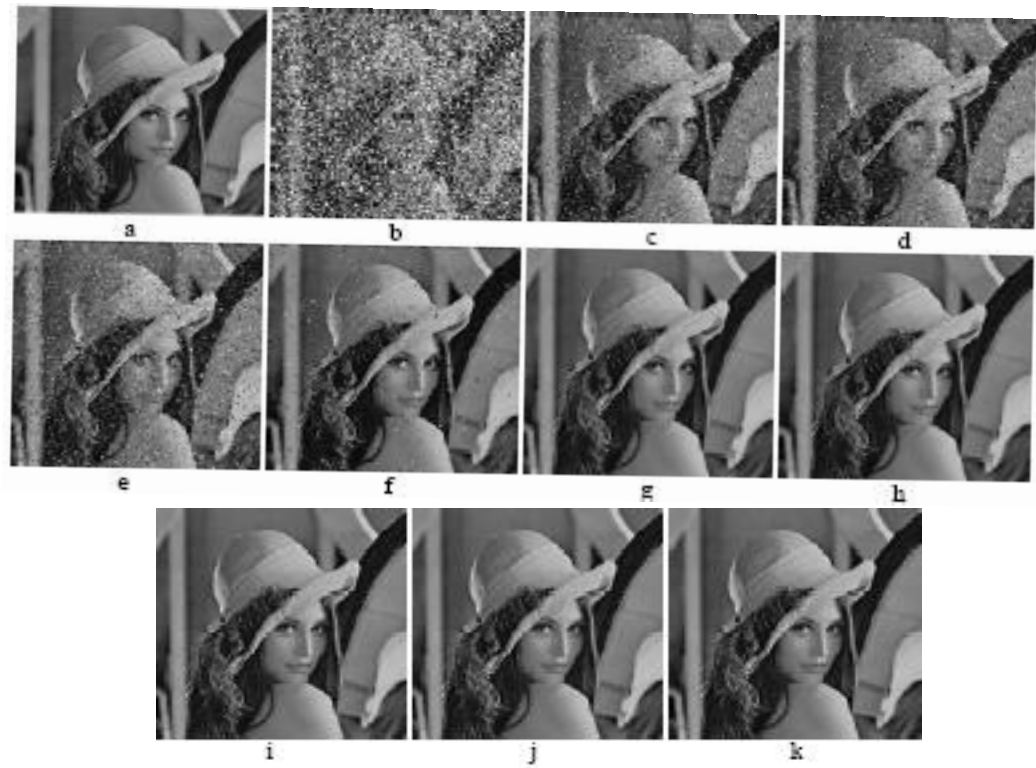

Fig 2: Results of different filters for Lena image corrupted by $50 \%$ noise. (a) Noise freeLena image, (b) Image corrupted by $50 \%$ impulse noise. (c) Output of SMF.(d) Output of PSMF. (e) Output of AMF. (f) Output of RAMF. (g) Output of DBA.(h) Output of Trilateral Filter using ROAD. (i) Output of REA. (j) Output of DWM.(k) Image restored by the proposed DPFMF
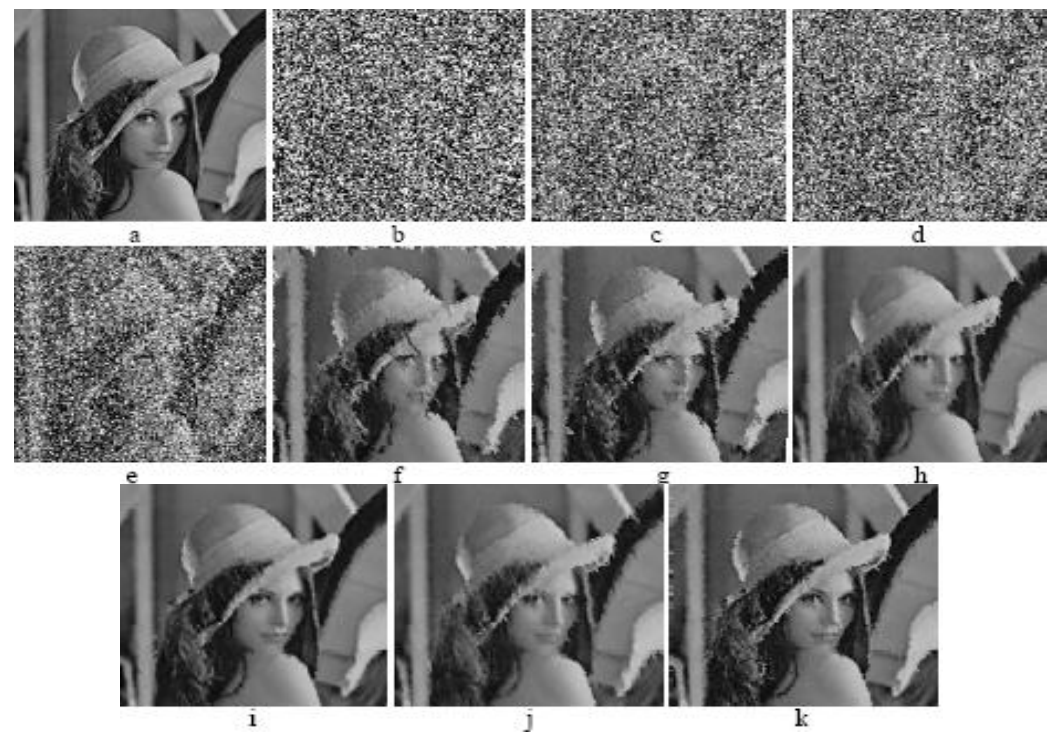

Fig 3: Results of different filters for Lena image corrupted by $80 \%$ noise.

(a) Noise free Lena image, (b) Image corrupted by $80 \%$ impulse noise. (c) Output of SMF. (d) Output of PSMF. (e) Output of AMF. (f) Output of RAMF. (g)

Output of DBA. (h) Output of Trilateral Filter using ROAD. (i) Output of REA. (j) Output of DWM. (k) Image restored by the proposed DPFMF 


\section{Conclusion}

In this paper, we have proposed a novel, fast, accurate and simple impulse-noise restoration method based on fast median filter output or by the value computed based on a unique criterion applied to the processed neighboring pixel values which has been further incorporated into the framework of a switching median filter. The extensive simulation results included in this work have demonstrated that the proposed DPFMF outperforms a number of well accepted median based filters that have been described in the literature. The DPFMF exhibits desirable robustness not only in suppressing the noise but also in gaining satisfactory image quality.

A common problem with the current nonlinear filtering techniques is that they perform excessive smoothing in texture areas. Further work will be in the direction of design of a new criterion that can distinguish between a textured and noisy neighborhood.

\section{Acknowledgements}

The authors are grateful to the anonymous reviewers for their valuable comments and suggestions.

\section{References}

[1] I.Pitas and A. N. Venetsanopoulos, Nonlinear Digital Filters Principles and Applications, Norwell, MA: Kluwer Publications, 1990

[2] J.Astola and P. Kuosmanen, Fundamentals of Nonlinear Digital Filtering. Boca Raton, CRC, 1997

[3] N.C.Gallagher, Jr. and G.L.Wise, "A Theoretical Analysis of the Properties of Median Filters," IEEE Trans. Acoustics, Speech and Signal Processing, ASSP-29 (Dec.), 1136-1141, 1981.

[4] T.A.Nodes and N.C.Gallagher, "Median Filters: Some Modifications and their properties," IEEE Trans. Acoustics, Speech and Signal Processing, ASSP-30 (Apr.), 739-746, 1987.

[5] E.Abreu, M.Lightstone, S.K.Mitra and K.Arakawa, "A New Efficient Approach for the Removal of Impulse Noise from Highly Corrupted Images," IEEE Trans. Image Processing, 5, (Jun.), 1012-1025, 1996.

[6] D.R.K.Brownrigg, "The Weighted Median Filter", Commun. Assoc. Comput. Machin, Vol.22, pp. 807-818,1984.

[7] Aug. O. Yli-Harja, J. Astola and Y. Neuvo, "Analysis of the Properties of Median and Weighted Median Filters Using Threshold Logic and tack Decomposition", IEEE Trans. Signal Processing, vol. 39(Feb.), pp. 395-410, 1991. 
[8] S.J. Ko and Y. H. Lee, "Center weighted median filters and their applications to image enhancement," IEEE Trans. Circuits Syst., vol. 38 (Sep.), pp. 984993, 1991.

[9] H.Hwang and R. A.Haddad, "Adaptive median filters: new algorithms and results,'IEEE Trans. Image Processing, vol. 4(Apr.), pp. 499- 502,1995.

[10] Z.Wang and D.Zhang, "Progressive Switching Median Filter for the Removal of Impulse Noise from Highly Corrupted Images," IEEE Trans. Circuits and Systems-II:Analog and Digital Signal Processing, vol. 46 (Jan), pp. 78-80, 1999.

[11] Raymond H. Chan, Chung-Wa Ho, and Mila Nikolova , "Salt-and-Pepper Noise Removal by Median-type Noise Detectors and Detail preserving Regularization", IEEE Trans. Image Processing, vol. 14 (Oct.), pp. 14791485, 2005.

[12] K. S. Srinivasan and D. Ebenezer, "A new fast and efficient decision-based algorithm for removal of high-density impulse noises," IEEE Signal Processing Letters, vol. 14 (Mar.), pp.189-192, 2007

[13] S. Schulte, M. Nachtegael, V. D. Witte, D. V. der Weken, and E. E. Kerre, "A fuzzy impulse noise detection and reduction method," IEEE Trans. Image Processing, 1153-1162, 15 (May),2006.

[14] A. Ben Hamza and Hamid Krim, "Image Denoising: A Nonlinear Robust Statistical Approach" IEEE Trans. signal processing, vol. 49 (Dec), pp. 3045-3054, 2001

[15] V.Jayaraj, D.Ebenezer, K.Aiswarya, “ High density salt and pepper noise removal in images using improved adaptive statistics estimation filter", International Journal of Computer Science and Network Security, vol. 9 (Nov.), pp.164 - 173, 2009.

[16] V.Jayaraj, D.Ebenezer, "A new switching based median filtering scheme and algorithm for removal of high density salt and pepper noise in images" EURASIP Journal on Advances in Signal Processing, Volume 2010 (2010), Article ID 690218, 11 pages, doi:10.1155/2010/690218,2010.

[17] S. Zhang and M. A. Karim, "A new impulse detector for switching median filters," IEEE Signal Processing Letters, vol. 9 (Nov.), pp. 360-363, 2002.

[18] E. Abreu, S.K. Mitra, 1995 "A signal-dependent rank ordered mean filter-a new approach for removal of impulses from highly corrupted images" International Conference on, Acoustics, Speech, and Signal Processing, ICASSP-95, 4, pp. 2371-2374,1995.

[19] Y. Dong and S. Xu, "A new directional weighted median filter for removal of randomvalued impulse noise," IEEE Signal Processing Letters, vol. 14 (Mar.), pp.193-196,2007.

[20] Crnojevic, V., Senk, V., Trpovski, Z." Advanced impulse Detection Based on pixel-wise MAD”, IEEE Signal Processing Letters, vol. 11 (Jul.), pp. 589 $-592,2004$. 
[21] R. Garnett, T. Huegerich, C. Chui and W.-J. He, ' Universal noise removal algorithm with an impulse detector," IEEE Transactions on Image Processing, vol. 14, pp. 1747-1754, 2005.

[22] Yiqiu Dong, Raymond Chan, and Shufang Xu, "A Detection Statistic for Random-Valued Impulse Noise" IEEE Trans. Image Processing, vol.16 (Apr.), pp.1112 - 1120,2007.

[23] N. I. Petrovic and V. Crnojevic, "Universal impulse noise filter based on genetic programming," IEEE Trans. Image Process., vol. 17 (Jul.), pp.11091120, 2008.

[24] Zhou Wang, Alan C. Bovik, Hamid R. Sheikh and Eero P. Simoncelli, "Image Quality Assessment:from Error Visibility to Structural Similarity" IEEE Trans. Image Processing, vol.13 (Apr.), pp. 600-612,2004.

[25] Madhu S. Nair, K.Revathy, Rao Tatavarti, "An Improved Decision Based Algorithm for Impulse Noise Removal" , Proceedings of International Congress on Image and Signal Processing -CISP IEEE Computer Society Press, Sanya, Hainan, China, Vol.1, pp.426- 431, May 2008. 